\title{
Multifocal Extensive Spinal Tuberculosis with Retropharyngeal Abscess
}

\author{
Farzana Shumy, ${ }^{1}$ Ahmad Mursel Anam², MA Jalil Chowdhury ${ }^{3}$, Md.Abul Kalam Azad ${ }^{2}$, Samsun Nahar ${ }^{2}$ \\ ${ }^{1}$ Medical Officer, ${ }^{2}$ Post Graduate Trainee and ${ }^{3}$ Professor, Department of Medicine, Bangabandhu Sheikh Mujib Medical University, \\ Shahbag, Dhaka-1000, Bangladesh.
}

\begin{abstract}
:
An unusual case of a young boy presenting with spinal tuberculosis involving cervical \& thoracic vertebrae, along with retropharyngeal abscess is reported. The patient presented with progressive quadriparesis, fever, night sweat and cervical lymphadenopathy. The lab studies confirmed tuberculosis and patient received anti-tubercular chemotherapy. After development of quadriparesis, spinal surgery was done. The post operative course was uneventful and the patient is on gradual neurological recovery.
\end{abstract}

[BSMMU J 2011; 4(2): 128-130]

\section{Introduction:}

Skeletal tuberculosis (TB) usually constitutes $1-3 \%$ of extrapulmonary TB, and spinal involvement is about half of them. ${ }^{1}$ TB of the cervical spine is so unusual that it comprises only $3 \%$ of cases of Pott's disease. In addition, retropharyngeal abscess as a presenting manifestation of tuberculosis of the cervical spine is rare. ${ }^{2}$ The most dangerous area for skeletal TB is the cervical region, due to the greater risk of quadriplegia and death. ${ }^{3}$ This article describes an extremely rare case of multifocal Pott's disease, along with retropharyngeal and paravertebral abscess.

\section{Case report:}

A 13-year-old boy had 71/2 months history of fever, anorexia, weight loss, and cervical lymphadenopathy with discharging sinus. Initially he was admitted in a tertiary care hospital, where, a CT scan of chest \& neck revealed “retropharyngeal, pre \& paravertebral abscess, multiple vertebral destruction, and cervical \& right paratracheal lymphadenopathy" (Figure 1). For the last 6 months, he was on supervised anti-TB chemotherapy on the basis of FNAC of cervical lymph node which revealed caseating granuloma. Despite getting Anti-TB drugs, and symptomatic improvement in terms of resolution of fever, improvement of appetite and weight, he developed neck pain along with quadriplegia, 5 days before presenting to us.

On admission, both his upper and lower limb power was $0 /$ 5 with complete sensory loss up to $\mathrm{C}_{2}$ and absent deep tendon reflexes with equivocal plantar response.

Address for Correspondence: Farzana Shumy, House \# 61/B, Road \# 6/A, Dhanmondi RA, Dhaka 1209, Bangladesh. E-mail: farzanashumy@hotmail.com

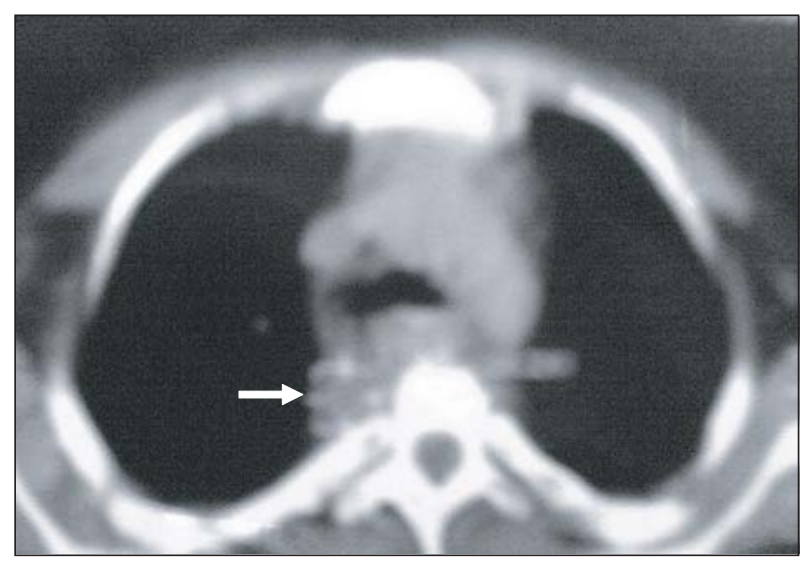

Fig.-1: CT scan of chest showing paravertebral abscess (arrow).

He had raised ESR ( $85 \mathrm{~mm}$ in the $1^{\text {st }}$ hour) with positive Mantoux Test (20 mm after 72 hours). MRI of cervical \& dorsal spine, done this time, showed " $\mathrm{C} 4$ \& D4 vertebral body heights are reduced with anterior wedging causing localized kyphotic deformity with epidural extension of the perivertebral mass resulting in cord compression. Disk space between C4-C5 and D4-D5 are obliterated. Perivertebral mass at craniovertebral junction to C5 level and D2 to D4-5 level with epidural extension present. Atlanto-axial subluxation with an intervening mass are causing further cord compression (Impression: Features are suggestive of tubercular spondylodiscitis at multiple levels with epidural mass causing cord compression)" (Figure 2).

All other biochemical investigations, including random blood sugar, were normal.

Histopathological examination of tissue from intervertebral disc and paravertebral region of $\mathrm{C}_{3}-\mathrm{C}_{4}$ region showed caseating granuloma. 


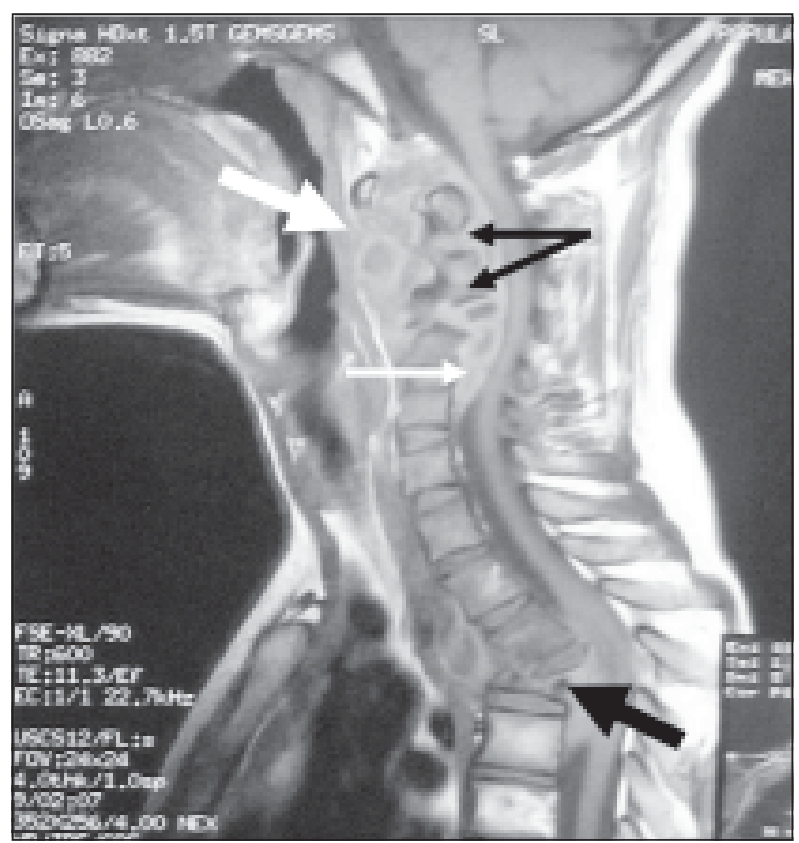

Fig.-2: MRI of cervical and upper dorsal spine showing destruction of cervical (thin black arrow) \& thoracic (thick black arrow) vertebrae, with epidural abscess causing cord compression (thin white arrow), along with retropharyngeal abscess (thick white arrow).

To manage quadriplegia, trans-oral and trans-cervical decompression of spinal cord at $\mathrm{C}_{2,3,4}$ level and fusion of loose graft was done. He was already on continuation phase of anti-tubercular chemotherapy.

After surgery, patient regained his upper limb power and sensory function of all limbs.

\section{Discussion:}

The retropharyngeal space is a potential space in the fascial planes between the prevertebral and buccopharyngeal fascia. ${ }^{2}$ Tuberculous retropharyngeal abscess is usually a consequence of chronic TB of the cervical spine, because pus spreads directly through the anterior longitudinal ligament. ${ }^{4}$

Our patient's presenting features, like neck pain and retropharyngeal abscess and collapse of cervical vertebrae, as demonstrated in CT scan, can be explained in this manner.

Primary focus in the lungs or the lymph nodes acts as a source for haematogenous dissemination, resulting in spinal tuberculosis. ${ }^{5}$ In this case; the patient had cervical lymphadenopathy as well as right paratracheal lymphadenopathy, either of which can be responsible.

Three recognized patterns of vertebral body involvement are paradiskal, anterior and central lesions. Paradiskal lesion, adjacent to the vertebral body, leads to narrowing of disc space. Central lesion targets the vertebral body without involving disc. Anterior lesions are subperiosteal lesions under the anterior longitudinal ligament. The central type spreads along the Batson's plexus of veins, while paradiskal infection spreads through the arteries. The anterior type of vertebral body tuberculosis results from the extension of the abscess beneath the anterior longitudinal ligament and periosteum. Pus spread over multiple vertebral segments, stripping the periosteum and anterior longitudinal ligament from anterior surface of vertebral bodies. MRI image shows abnormal signal involving multiple vertebral segments. ${ }^{6}$ These, probably, were the processes, in which multiple vertebral involvement, along with perivertebral abscess in both cervical and thoracic region, took place in our patient, although extensive bone destruction involving multiple vertebrae is an uncommon finding in tuberculous spondylitis.

Spinal tuberculosis likes to target the thoracic and the lumbar spine; involvement of the cervical region and sacrum is less common. ${ }^{7}$ As cross-sectional diameter of the spinal canal is relatively smaller than the diameter of the cervical cord; neurologic deficits are easier and earlier, if cervical spine is involved. Neurologic symptoms can manifest by any of the following process: subluxation of vertebrae, impingement of bone, disc, and abscess on the spinal cord or nerve root, local inflammatory response, and tuberculous vasculitis. ${ }^{1}$

CT scan of our patient demonstrated, spinal cord compression due to collapse of vertebra along with epidural extension of perivertebral mass, which explains the neurological deficit in our patient.

Spinal involvement with skip lesion has also been found in Brucellosis. Histopathology usually demonstrates noncaseating granulomatous tissue. Spinal brucellosis may be distinguished from tuberculosis by MRI also. In brucellosis, the vertebral body is usually morphologically intact despite evidence of osteomyelitis and the disc is moderately reduced in size despite evidence of involvement. ${ }^{8}$

Surgical intervention in Pott's disease is indicated if there is neurologic deficit, spinal deformity with instability or pain, no response to anti-TB chemotherapy (evidenced by continuing progression of kyphosis or instability) and large paraspinal abscess. ${ }^{9}$ 


\section{References:}

1. Idris SK, Abdulkadir AY. Tuberculous Retropharyngeal Abscess with Posterior Mediastinal Extension and Quadriplegia in A 13-Year-Old Nigerian Girl. Int $J$ of Pediatr Otorhinolaryngol Extra. 5 (2010): 118-120.

2. Al Soub H. Retropharyngeal Abscess Associated with Tuberculosis of the Cervical Spine. Tuber LungDis 1996;77: 563-565.

3. Turgut M. Multifocal Extensive Spinal Tuberculosis (Pott's Disease) Involving Cervical, Thoracic and Lumbar Vertebrae. Br J Neurosurg 2001; 15(2): 142-146.

4. Mizumura K, Machino T, Sato Y, Ooki T, Hayashi K, Nakagawa Y et al. Tuberculous Retropharyngeal Abscess Associated with Spinal Tuberculosis Well Controlled by Fine-needle Aspiration and Anti-tuberculous Chemotherapy. Inter Med 2010; 49: 1155-1158.

5. Chauhan A, Gupta BB. Spinal Tuberculosis. JIACM 2007; 8(1): 110-114.

6. Khattry N, Thulkar S, Das A, Khan SA, Bakhshi S. Spinal Tuberculosis Mimicking Malignancy: Atypical Imaging Features. Indian J Paediatr 2007;74(3):297-298.

7. Shanley DJ. Tuberculosis of the Spine: Imaging Features. AJR 1995;164:659-664.

8. Coskun E, Süzer T, Yalçin N, Tahta K. Spinal Extradural Compression caused by Granuloma of Brucellosis. Scand J Infect Dis 1998;30(3):311-312.

9. Watts HG, Lifeso RM. Current Concepts Review - Tuberculosis of Bones and Joints. J Bone Joint Surg Am. 1996;78(2):288-99. 